\title{
Prospective study of gefitinib readministration after chemotherapy in advanced non-small cell lung cancer patients who previously responded to gefitinib
}

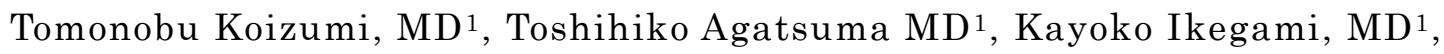
Toshiro Suzuki MD¹, Takashi Kobayashi MD¹, Shintaro Kanda MD¹, Sumiko Yoshikawa MD르, Keishi Kubo MD ${ }^{1}$, Takayuki Shiina MD ${ }^{1}$, Keiichirou Takasuna $\mathrm{MD}^{2}$, Akemi Matsuo MD³, Muneharu Hayasaka MD4, Miwa Morikawa MD ${ }^{5}$, Shingo Ameshima MD ${ }^{5}$

1) Respiratory Center, Shinshu University School of Medicine, Matsumoto, Japan

2) Division of Thoracic Surgery, Ina Chuo Hospital, Ina, Japan

3) Respiratory Division, Shinonoi Koseiren Hospital, Nagano Japan

4) Respiratory Division, National Chushin Matsumoto Hospital, Matsumoto, Japan

5) Department of Respiratory Medicine, University of Fukui, Fukui, Japan

Corresponding Author: Tomonobu Koizumi, MD, PhD,

Comprehensive Cancer Center, Division of Clinical Oncology, Shinshu University Hospital, 3-1-1 Asahi Matsumoto, 390-8621 Japan

Phone: +81-263-37-2631 Fax:+81-263-36-3722

Email: tomonobu@shinshu-u.ac.jp

Running title; Gefitinib readministration for NSCLC in previous responders Number of Words; 3887 


\section{Disclusures}

There is no conflict of interest in relation to this study in all authors.

\section{MicroAbstract}

The present study was designed to prospectively evaluate the clinical efficacy of gefitinib readministration in patients with advanced non-small cell lung cancer who responded well to initial gefitinib and followed by cytotoxic chemotherapy. 20 subjects were enrolled and three and six patients achieved partial response and stable disease. These findings provide valuable information for the management of previous gefitinib responders. 


\section{ABSTRACT}

Introduction: Salvage treatment for acquired resistance to epidermal growth factor receptor tyrosine kinase inhibitor (EGFR-TKI) in patients with non-small cell lung cancer (NSCLC) is a matter of clinical concern. Several retrospective reports have indicated the usefulness of EGFR-TKI readministration, however, there have been few prospective studies.

Materials and Methods: This study was designed to prospectively evaluate the clinical efficacy of gefitinib readministration in patients with advanced or metastatic NSCLC who responded well to initial gefitinib treatment. Subjects received at least one regimen of cytotoxic chemotherapy after progressive disease (PD) with the initial gefitinib therapy. Gefitinib administration $(250 \mathrm{mg} / \mathrm{day})$ was started after $\mathrm{PD}$ with the previous chemotherapeutic regimen. The primary endpoint in the present study was the response rate (RR).

Results : Twenty patients were enrolled between April 2007 and May 2011. Three patients achieved partial response and 6 showed stable disease. Thus, the overall $\mathrm{RR}$ and disease control rate of gefitinib readministration were $15 \%$ (95\% confidence interval (CI): $3.2-37.9)$ and $45 \%(95 \%$ CI: $23.1-68.5)$, respectively. Median progression-free survival and overall survival from the start of gefitinib readministration were $2.0(95 \% \mathrm{CI}: 0.9-3.1)$ and 12.0 (95\% CI: 8.0-16.0) months, respectively.

Conclusion: These results suggest that gefitinib readministration may be an option, albeit with a low response rate and short progression-free survival, for patients 
who responded well to initial gefitinib followed by systemic chemotherapy. These findings provide valuable information for the management of previous gefitinib responders.

Key words ; EGFR-TKI, re-charrenge, Gefitinib responder, NSCLC, EGFR mutation, chemotherapy 


\section{Introduction}

Gefitinib, an oral small molecule agent that acts as an epidermal growth factor receptor tyrosine kinase inhibitor (EGFR-TKI), is the first molecular targeted agent to be approved for the treatment of patients with advanced non-small cell lung cancer (NSCLC). The extremely high response rate for gefitinib is associated with the presence of active $E G F R$ mutations in tumor cells, such as in-frame deletions in exon 19 or point mutations in exon 21 (e.g., L858R). 1-7 Two phase III trials comparing chemotherapy to gefitinib in a first-line setting demonstrated that gefitinib could produce improved progression-free survival (PFS) compared to chemotherapy in patients harboring EGFR-activating mutations.6,7 EGFR-TKI in the second line after chemotherapy showed similar activity as the first line in sensitive patients with EGFR mutations.4,8,9 Furthermore, as second-line chemotherapy, a phase III study demonstrated superior PFS with gefitinib than docetaxel for unselected patients in Korea. ${ }^{10}$. Thus, gefitinib is useful for the firstand second-line treatment of NSCLC.

Tumors initially responded to EGFR-TKI, but most patients with NSCLC eventually showed a progressive disease (PD). Although several possible mechanisms of secondary acquired $E G F R$ mutations 11,12 or other unrelated pathways to $E G F R$ genotypes ${ }^{13}$ were reported, successful strategies for overcoming the resistance to EGFR-TKI have yet to be established.14-16 Thus, the development of a novel and possible therapeutic strategy for patients with resistance to EGFR-TKI is an important clinical issue in medical oncology. 
Several case reports and retrospective clinical analysis have indicated that the patients with NSCLC who responded to gefitinib in the initial treatment were successfully controlled by readministration of gefitinib. ${ }^{17,18}$ However, there were a few prospective studies to evaluate the efficacy of gefitinib readministration in patients with NSCLC. ${ }^{14}$ In the present study, we prospectively investigated the efficacy of gefitinib readministration in 20 patients with NSCLC who initially responded well to gefitinib. The subjects received at least a cytotoxic chemotherapy after progression to initial gefitinib. Then, the enrolled patients were treated with readministration of gefitinib after progression to the chemotherapy.

\section{Patients and methods}

Subjects with histologically or cytologically confirmed NSCLC and recurrent or metastatic NSCLC (stage IV) were enrolled in this study. Patients with the following treatment histories were eligible: 1) responded to initial gefitinib treatment (complete response (CR), partial response (PR), or stable disease (SD) over 6 months), 2) documented PD to the initial gefitinib according to the Response Evaluation Criteria in Solid Tumors (RECIST) guidelines ${ }^{20}, 3$ ) and then received at least one subsequent cytotoxic chemotherapy regimen. Other eligibility criteria included Eastern Corporative Oncology Group performance status (PS) 0-3, measurable tumor lesion, and adequate organ functions. Brain metastases were also allowed if they were asymptomatic or controlled by supportive care. However, patients who could not continue administration of gefitinib during the initial 
therapy due to unresolved toxicities were excluded from the study.

Objective tumor response during gefitinib readministration was evaluated by computed tomography (CT) every 4 weeks in accordance with the RECIST guidelines. Chest radiographs were also taken every 2 weeks after initiation of gefitinib readministration. In cases with suspicious findings for $\mathrm{PD}$ on chest radiographs, an additional evaluation by $\mathrm{CT}$ was performed. In the present study, the minimum interval for determining SD during gefitinib readministration was defined as at least 8 weeks. Enrolled patients were retreated with gefitinib (250 mg/day) with no dose reduction unless toxicities above grade 3 developed. Treatment was continued until PD, intolerable toxicity, or withdrawal of consent. In unacceptable events of toxicity defined over grade 3 , gefitinib was stopped until the toxicity resolved and improved to below grade 3 . No dose reduction was permitted, but a modification such as administration every two days, was allowed when re-treatment with gefitinib. Study protocol was approved by the institutional review boards of each participating hospital. Written informed consent was obtained from each patient prior to enrolment.

\section{Statistical analysis}

Objective response rate $(\mathrm{RR})$ with gefitinib readministration was taken as the primary endpoint in the present study. Simon's two-stage MiniMax design was used to determine the sample size. We set $R R=25 \%$ in enrolled patients and a rate of $5 \%$ as the lower limit of interest, with $\alpha=0.1$ and $\beta=0.1$. The estimated minimum sample size for was 20 cases. Secondary endpoints were disease control rate (DCR), 
PFS, overall survival (OS), and toxicity. DCR was defined as the sum of the rate of RR plus SD. PFS and OS probabilities after the initiation of gefitinib readministration were based on Kaplan-Meier method. Reevaluation of the protocol was proposed if no-responders to gefitinib readministration were included in the first 13 enrolled subjects. In the present study, several factors potentially affecting the differences between patients achieving $\mathrm{PR}+\mathrm{SD}$ and $\mathrm{PD}$ were compared. Categorical variables were compared using Fisher's exact or the $\chi^{2}$ test. The duration of gefitinib treatment was calculated from the date of initiation of gefitinib to the date of its withdrawal. Adverse events were graded according to the National Cancer Institute Common Terminology Criteria for Adverse Event version 3.0.21 Confidence intervals were calculated at the $95 \%$ level $[95 \%$ confidence interval (CI)]. Dr. SPSS software (SPSS, Inc., Chicago, IL) was used for the analyses. 


\section{Results}

\section{Patient characteristics}

Twenty patients were enrolled between April 2007 and May 2011. The clinical characteristics are summarized in Table 1 . The median age of patients was 61 years (range: $41-81$ years). Seventeen patients were female (85\%), 18 were non-smokers (90\%), and all patients had adenocarcinoma. One patient initially received non-platinum chemotherapy (docetaxel) because of advanced age, while the other patients were treated with platinum doublet regimens including cisplatin (CDDP) plus docetaxel or carboplatin (CBDCA) plus paclitaxel, etc. Three patients were treated with gefitinib as first-line therapy. These patients then received CDDP + docetaxel, CDDP + pemetrexed, and CBDCA + paclitaxel, respectively. In most cases, initial gefitinib therapy was performed in the second line (9 cases) or third-line ( 7 cases)(Table 2$)$. Of the total of 20 cases, 17 (85\%) had PR and $3(15 \%)$ had SD with the initial gefitinib treatment. The mean treatment interval was 13.9 \pm 8.7 in patients with $\mathrm{PR}$ and $8.0 \pm 3.5$ months in patients with SD. All patients had received various cytotoxic chemotherapeutic regimens before enrollment. Two and three regimens of cytotoxic chemotherapies were serially used in two patients, respectively. The remaining 16 patients received one regimen between initial and readministration of gefitinib. Among 16 cases, two patients were treated with CBDCA + paclitaxel, one with $\mathrm{CDDP}+$ pemetrexed and most cases received a single-agent chemotherapeutic regimen including docetaxel, pemetrexed, and S-1 et al. The best response to previous chemotherapy before gefitinib 
readministration included two cases of $\mathrm{PR}, 11$ cases of SD and 7 cases of PD.

\section{Tumor Response and survival}

Responses were evaluated for all patients (Table 3). Three patients achieved PR and 6 patients showed SD. Thus, the overall RR and DCR of gefitinib readministration were $15 \%(95 \%$ CI: $3.2-37.9)$ and $45 \%$ (95\% CI: 23.1-68.5), respectively. Median PFS and OS from the start of gefitinib readministration were $2.0(95 \%$ CI; 0.9-3.1)(Fig. 1A) and 12.0 (95\%CI: 8.0-16.0) months (Fig. 1B), respectively. Figure 2 shows OS from the initial therapy; the median OS was 48.3 months $(96 \%$ CI: $35.3-61.3)$.

In 10 patients, $E G F R$ mutation status was determined at initial diagnosis of NSCLC or before enrollment in the study (summarized in Table 4). There were no patients with a T790M point mutation in exon 20 of the $E G F R$ gene. Four patients had sensitive mutations (L858R) just before gefitinib readministration. However, the response to gefitinib readministration was $\mathrm{SD}$ in one patient and $\mathrm{PD}$ in three patients. Thus, these were not always related to a good response. In terms of $E G F R$ status in three responders to gefitinib readministration, wild type in one case and unknown in two cases. In addition, we analyzed the association of responses to the second course of gefitinib therapy with the period between first and second courses of gefitinib treatment and responses to cytotoxic chemotherapies administered subsequently after the first gefitinib failed. There was no correlation between response to the second gefitinib and the length of interval of gefitinib treatment $(6.8 \pm 4.3$ months in over SD vs. $6.2 \pm 2.7$ months in PD). 


\section{Adverse Events}

The toxicities were assessed in all 20 patients and were compared with those seen during the initial therapy (Table 5). The most common adverse event was grade $1 / 2$ skin toxicity. Grade 3 skin toxicity occurred in three patients in the initial therapy, and in one patient during gefitinib readministration. One patient developed grade 3 hepatotoxicity two weeks after gefitinib readministration. The patient showed similar hepatotoxicity (Grade 3) during the initial gefitinib treatment. The gefitinib therapy was continued with every two days in this case but PD occurred 1.5 months after the start of gefitinib readministration. No interstitial lung disease was observed, and no other patients discontinued gefitinib due to toxicity during the period of readministration. 


\section{Discussion}

We conducted a prospective study to evaluate the efficacy of gefitinib readministration in patients with a previous response to initial gefitinib therapy. Subjects treated with cytotoxic chemotherapies between the initial and second course of gefitinib therapy were enrolled in the present study. Fifteen percent of patients had PR to gefitinib readministration, although the interval of response was transient.

Several retrospective studies or case reports suggested that gefitinib readministration was useful in patients who responded to initial gefitinib.17-19 Recently, Asahina et al ${ }^{14}$. reported results of the first prospective phase II study with a design similar to that of the present study. They reported that none of 16 patients responded to gefitinib readministration, but DCR was $44 \%$ in their study. As 4 patients achieved long-term SD ( $>6$ months), they emphasized that some of the selected patients could respond to gefitinib readministration. We experienced a response rate of $15 \%$ in the present study, but the DCR was similar to that in the report of Asahina et al ${ }^{14}$. Based on our results and those of other reports ${ }^{14,17-19}$, gefitinib readministration could be a potential therapeutic option in patients with NSCLC who responded to the initial course of gefitinib treatment followed by systemic chemotherapy.

PFS was evaluated as a secondary endpoint in the present study. The median PFS was 2.0 months, which was also identical to that (2.7 months) in the study of Asahina et al ${ }^{14}$. Patients in whom gefitinib readministration was successful (SD + 
PR) showed a better PFS (4 months) than those with PD (1.5 months) in the present study. Thus, optimal patient selection is still unclear, but we would like to emphasize that gefitinib readministration may show clinical benefit in some patients who previously responded well to gefitinib.

The insertion of cytotoxic chemotherapies before gefitinib readministration could influence the response to second gefitinib administration. Several retrospective studies regarding gefitinib readministration have shown that a prolonged gefitinib-free interval was a predictive factor for a favorable clinical result. ${ }^{18,19}$ In addition, in cases with switching to another EGFR-TKI (erlotinib) after gefitinib failure, the EGFR-TKI-free interval with insertion of cytotoxic chemotherapy between gefitinib and erlotinib was associated with longer PFS after the initiation of erlotinib 22 . It is demonstrated that tumor tissues in NSCLC could include histologically heterogeneous components and that a detection of positive or resistant EGFR mutated tumor cells could be varied with different tumor sites in certain cases 23,24 . Thus, cytotoxic chemotherapy administered after a resistance to the initial EGFR-TKI may modify the heterogeneous tissue distribution in sensitive or resistant cells. We speculated that if the proportion of tissue sensitive to EGFR-TKI would change to be dominant after cytotoxic chemotherapy, and gefitinib readministration could be beneficial in such cases. However, no significant differences were observed in the present study regarding the EGFR-TKI-free intervals between patients with and without PR $+\mathrm{SD}$, consistent with the findings of the prospective study by Asahina et al ${ }^{14}$. 
Measurement of $E G F R$ mutation status is useful for selection of sensitive tumors and is generally performed at the initial diagnosis of NSCLC. However, our study had the limitation that EGFR-related biomarkers were not assessed for all enrolled subjects as testing for $E G F R$ mutation was not available at the start of this study. Although the sample sizes were too small in the present study, we found that detection of sensitive $E G F R$ mutations just before gefitinib readministration was unrelated to clinical benefit. In these EGFR mutation-positive cases, the mechanism of resistance to gefitinib readministration may be related to other molecular pathways than the presence of T790M point mutation. Further investigations are required to clarify the mechanisms of sensitivity and resistance to gefitinib readministration for optimal patient selection.

A number of second-generation EGFR inhibitors are being studied, some of which show the potential to overcome or prevent the development of acquired resistance in patients previously treated with gefitinib or erlotinib.15,16. However, the possibility of clinical application is still in the preliminary stages. Thus, at present, data on the efficacy for overcoming acquired resistance in patients who previously responded well to first-generation EGFR-TKI are lacking. Subsequent gefitinib readministration after some interval could be a novel therapeutic strategy in patients with acquired resistance to initial gefitinib treatment.

\section{Conclusion}

Gefitinib readministration following a certain interval should be considered as one of the therapeutic options for selected patients who showed a good response to 
initial gefitinib treatment. New therapeutic approaches for identifying molecular markers affecting resistance to EGFR-TKI are required to break through the development of resistance to EGFR-TKI.

\section{Clinical Practice Points}

Gefitinib, an oral small molecule agent that acts as an epidermal growth factor receptor tyrosine kinase inhibitor (EGFR-TKI), is the first molecular targeted agent to be approved for the treatment of patients with advanced non-small cell lung cancer (NSCLC). Gefitinib is useful for the first- and second-line treatment of NSCLC. Tumors initially responded to gefitinib, but the most patients with NSCLC eventually showed disease progression. Optimal strategies for overcoming the resistance to gefitinib have yet to be established. The present study was designed to prospectively evaluate the clinical efficacy of gefitinib readministration in patients who responded well to initial gefitinib and followed by cytotoxic chemotherapy. The present study showed favorable response and disease control rate, although number of enrolled patients was small. The results of the present study suggested that gefitinib readministration may be a reasonable option in patients with NSCLC who responded well to initial gefitinib followed by systemic chemotherapy. These findings provide valuable information for the management of previous gefitinib responders. 


\section{Acknowledgments}

We thank the patients and their families for their support and participation in this study. 
Figure 1

Kaplan-Meier plot of progression-free survival (A) and overall survival (B) after initiation of gefitinib readministration. The median period of progression-free survival was 2 months (95\% CI: $0.9-3.1$ months) and overall survival 12 months (95\% CI: 8 - 16 months).

Figure 2

Kaplan-Meier plot of overall survival after the initial treatment for all eligible patients. Median OS was 48.3 months (95\% CI: $35.3-61.3$ months). 


\section{Refferences}

1. Lynch TJ, Bell DW, Sordella R, et al. Activating mutations in the epidermal growth factor receptor underlying responsiveness of non-small-cell lung cancer to gefitinib. N Engl J Med 2004;350: 2129-39.

2. Paez JG, Jänne PA, Lee JC, et al. EGFR mutations in lung cancer: correlation with clinical response to gefitinib therapy. Science 2004; 304: 1497-500.

3. Inoue A, Suzuki T, Fukuhara T, et al. Prospective Phase II Study of Gefitinib for Chemotherapy-Naïve Patients With Advanced Non-Small-Cell Lung Cancer With Epidermal Growth Factor Receptor Gene Mutations. J Clin Oncol 2004; $24: 3340-6$.

4. Morita S, Okamoto I, Kobayashi K, et al. Combined survival analysis of prospective clinical trials of gefitinib for non-small cell lung cancer with EGFR mutations. Clin Cancer Res 2009; 15:4493-8.

5. Asami K, Koizumi T, Hirai K,et al. Gefitinib as first-line treatment in elderly epidermal growth factor receptor-mutated patients with advanced lung adenocarcinoma: results of a Nagano Lung Cancer Research Group Study. Clin Lung Cancer 2011; in press

6. Maemondo M, Inoue A, Kobayashi K, et al. Gefitinib or chemotherapy for non-small-cell lung cancer with mutated EGFR. N Engl J Med 2010; 362:2380-8.

7. Mitsudomi T, Morita S, Yatabe Y, et al. Gefitinib versus cisplatin plus docetaxel in patients with non-small-cell lung cancer harbouring mutations of the epidermal growth factor receptor (WJTOG3405): an open label, randomised 
phase 3 trial. Lancet Oncol 2010;2:121-8.

8. Wu JY, Yu CJ, Shih JY, et al. Influence of first-line chemotherapy and EGFR mutations on second-line gefitinib in advanced non-small cell lung cancer. Lung Cancer 2010;67;348-54.

9. Douillard JY, Shepherd FA, Hirsh V, et al. Molecular predictors of outcome with gefitinib and docetaxel in previously treated non-small-cell lung cancer: data from the randomized phase III INTEREST trial. $J$ Clin Oncol 2010; 28:744-52.

10. Lee DH, Park K, Kim JH, et al. Randomized Phase III trial of gefitinib versus docetaxel in non-small cell lung cancer patients who have previously received platinum-based chemotherapy. Clin Cancer Res 2010; 16:1307-14.

11. Engelman JA, Zejnullahu K, Mitsudomi T, et al. MET amplification leads to gefitinib resistance in lung cancer by activating ERBB3 signaling. Science 2007; $316 ; 1039-43$

12. Kobayashi S, Boggon TJ, Dayaram T, et al. EGFR mutation and resistance of non-small-cell lung cancer to gefitinib. N Engl J Med 2005; 352:786-92.

13. Yano S, Wang W, Li Q, et al. Hepatocyte growth factor induces gefitinib resistance of lung adenocarcinoma with epidermal growth factor receptor-activating mutations. Cancer Res 2008; 68:9479-87.

14. Asahina H, Oizumi S, Inoue A, et al. Phase II study of gefitinib readministration in patients with advanced non-small cell lung cancer and previous response to gefitinib. Oncology 2010; 79:423-9.

15. Giaccone G, Wang Y. Strategies for overcoming resistance to EGFR family 
tyrosine kinase inhibitors. Cancer Treat Rev 2011; Feb 28. [Epub ahead of print]

16. Riely GJ. Second-generation epidermal growth factor receptor tyrosine kinase inhibitors in non-small cell lung cancer. $J$ Thorac Oncol 2008; 3: S146-S149.

17. Yano S, Nakataki E, Ohtsuka S, et al. Retreatment of lung adenocarcinoma patients with gefitinib who had experienced favorable results from their initial treatment with this selective epidermal growth factor receptor inhibitor: a report of three cases. Oncol Res 2005; 15:107-11.

18. Tomizawa Y, Fujita Y, Tamura A, et al. Effect of gefitinib re-challenge to initial gefitinib responder with non-small cell lung cancer followed by chemotherapy. Lung Cancer 2010; 68:269-72.

19. Yokouchi H, Yamazaki K, Kinoshita I, et al.Clinical benefit of readministration of gefitinib for initial gefitinib-responders with non-small cell lung cancer. BMC Cancer 2007; 7:51.

20. Therasse P, Arbuck SG, Eisenhauer EA, et al. New guidelines to evaluate the response to treatment in solid tumors. European Organization for Research and Treatment of Cancer, National Cancer Institute of the United States, National Cancer Institute of Canada. J Natl Cancer Inst 2000; 92: 205-16.

21.Common Terminology Criteria for Adverse Events (CTCAE) Version 3.0 [National Cancer Institute Web site]. May 22, 2003. Available at: http://ctep.cancer.gov/protocolDevelopment/electronic_applications/ctc.htm. Accessed March 1, 2011. 
22. Hata A, Katakami N, Yoshioka H, et al. Erlotinib after gefitinib failure in relapsed non-small cell lung cancer: Clinical benefit with optimal patient selection. Lung Cancer 2011; Apr 27. [Epub ahead of print]

23. Bozzetti C, Tiseo M, Lagrasta C, et al. Comparison between epidermal growth factor receptor (EGFR) gene expression in primary non-small cell lung cancer (NSCLC) and in fine-needle aspirates from distant metastatic sites. $J$ Thorac Oncol 2008; 3:18-22.

24. Ushiki A, Koizumi K, Kobayashi N, et al. Genetic heterogeneity of EGFR mutation in pleomorphic carcinoma of the lung- response to gefitinib and clinical outcome - Jap J Clin Oncol 2009; 39:267-70. 
Table 1

\section{Patients Characteristics}

Mean Age 61 (41-81 years)

$$
\mathrm{M} ; \mathrm{F} \quad 3 ; 17
$$

Performance Status

$\begin{array}{ll}0 & 9 \\ 1 & 5 \\ 2 & 5 \\ 3 & 1\end{array}$

Histology

Adenocarcinoma 20

Smoking History

Never smoker 18

Smoker 2 (7 and 30 Pack Year, respectively)

Chemotherapy Regiems Before Initial Gefitinib ( $n=17)$

Platinum doublets 16

Non-platinum $\quad 1$

Previous Response to Initial Gefitinib and Mean Treatment Intervals ( $=20)$

$\begin{array}{lrrll}\text { Partial Response } & 17 & 13.9 \pm 8.7 & \text { months (range } & 6-38) \\ \text { Stable Disease } & 3 & 8.0 \pm 3.5 & \text { months (range } & 6-12)\end{array}$

Treatment Intervals of Initial Gefitinib $(n=20)$

$\begin{array}{rlcc}\text { Mean } & 13.0 & \pm & 8.3 \text { (months) } \\ 6-12 & \text { months } & & 13 \text { cases } \\ 13-16 & \text { months } & & 3 \text { cases } \\ 17-24 & \text { months } & & 3 \text { cases } \\ 25-38 & \text { months } & & 1 \text { cases }\end{array}$

Best Response to Previous Cytotoxic Chemotherapyies Before Gefitinib Readministration

\begin{tabular}{lc} 
Partial Response & \multicolumn{1}{c}{2} \\
Stable Disease & 11 \\
Progressive Disease & 7
\end{tabular}


Table 2

Therapy Lines of Initial and Readministration of Gefitinib

\begin{tabular}{ccc} 
Lines & Initial & Readministration \\
\hline 1 st & 3 & \\
2 nd & 9 & \\
3 rd & 7 & 3 \\
4 th & 1 & 8 \\
5 th & & 6 \\
6 th & & 1 \\
7 th & & 2 \\
\hline
\end{tabular}


Table 3

Response to gefitinib readministration

\begin{tabular}{lc}
\hline \multicolumn{1}{c}{ Response } & No. of patients \\
\hline Complete response & 0 \\
Partial response & 3 \\
Stable disease & 6 \\
Progressive disease & 11 \\
& \\
Response rate (\%) & $15(95 \% \mathrm{Cl}: 3.2-37.9)$ \\
Disease control rate (\%) & $45(95 \% \mathrm{Cl}: 23.1-68.5)$ \\
\hline
\end{tabular}


Table 4

EGFR mutation status in available subjects

\begin{tabular}{clcccc}
\hline & Initial & & \multicolumn{2}{c}{ Readministration } \\
$\begin{array}{c}\text { EGFR } \\
\text { mutation } \\
\text { status }\end{array}$ & \multicolumn{1}{c}{ Sample } & Response & $\begin{array}{c}\text { EGFR } \\
\text { mutation } \\
\text { status }\end{array}$ & Sample & Response \\
ND & & PR & Wild type & Cerebrospinal & fluid \\
ND & & PR & L858R & Pleural effusion & SD \\
19 deletion & Pleural effusion & PR & ND & PD \\
L858R & Cervical LN & PR & ND & PD \\
L858R & Cervical LN & SD & L858R & Cervical LN & PD \\
L858R & Primary tumor & PR & ND & & PD \\
19 deletion & Primary tumor & PR & ND & & PD \\
L858R & Primary tumor & PR & L858R & Pleural effusion & PD \\
L858R & Primary tumor & PR & ND & & SD \\
L858R & Pleural effusion & PR & L858R & Pleural effusion & PD \\
\hline
\end{tabular}

ND; not done, LN; lymphnodo,

PR; partial response, SD; stable disease, PD; progressive disease 


\section{Table 5}

Adverse events during readministration and initial therapy of gefitinib

\begin{tabular}{cccccccc}
\hline & & \multicolumn{5}{c}{ Numbers in each Grade } & \\
\cline { 3 - 6 } & Toxicities & 0 & 1 & 2 & 3 & 4 & $\begin{array}{c}\% \geqq \\
\text { Garde 3 } \\
\text { toxicities }\end{array}$ \\
& & & & & & & \\
\hline \multirow{2}{*}{ Skin } & Readministration & 8 & 7 & 4 & 1 & 0 & 5 \\
& Initial therapy & 3 & 8 & 6 & 3 & 0 & 15 \\
\hline \multirow{2}{*}{ Diarrhea } & Readministration & 18 & 2 & 0 & 0 & 0 & 0 \\
& Initial therapy & 17 & 1 & 2 & 0 & 0 & 0 \\
\hline \multirow{2}{*}{ Hepatic } & Readministration & 19 & 0 & 0 & 1 & 0 & 5 \\
& Initial therapy & 17 & 2 & 0 & 1 & 0 & 5 \\
\hline
\end{tabular}



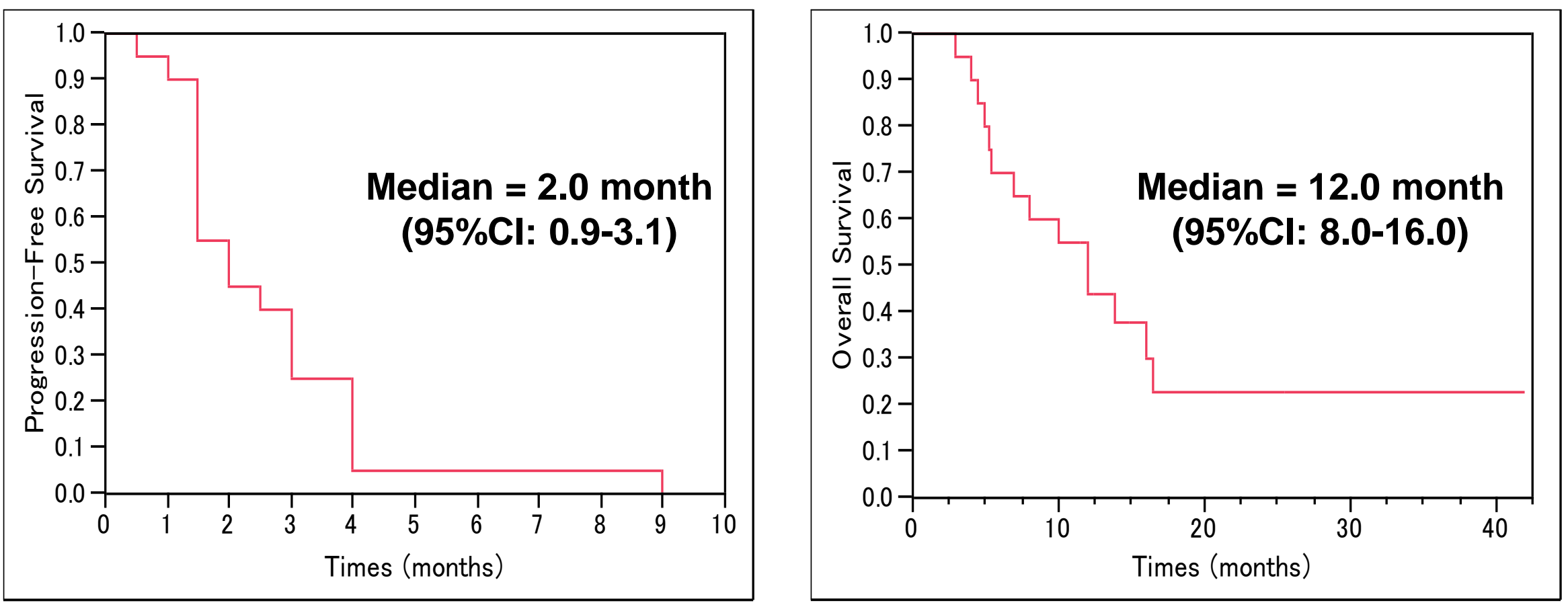

Figure $1 \mathrm{~A}$

Figure 1B 


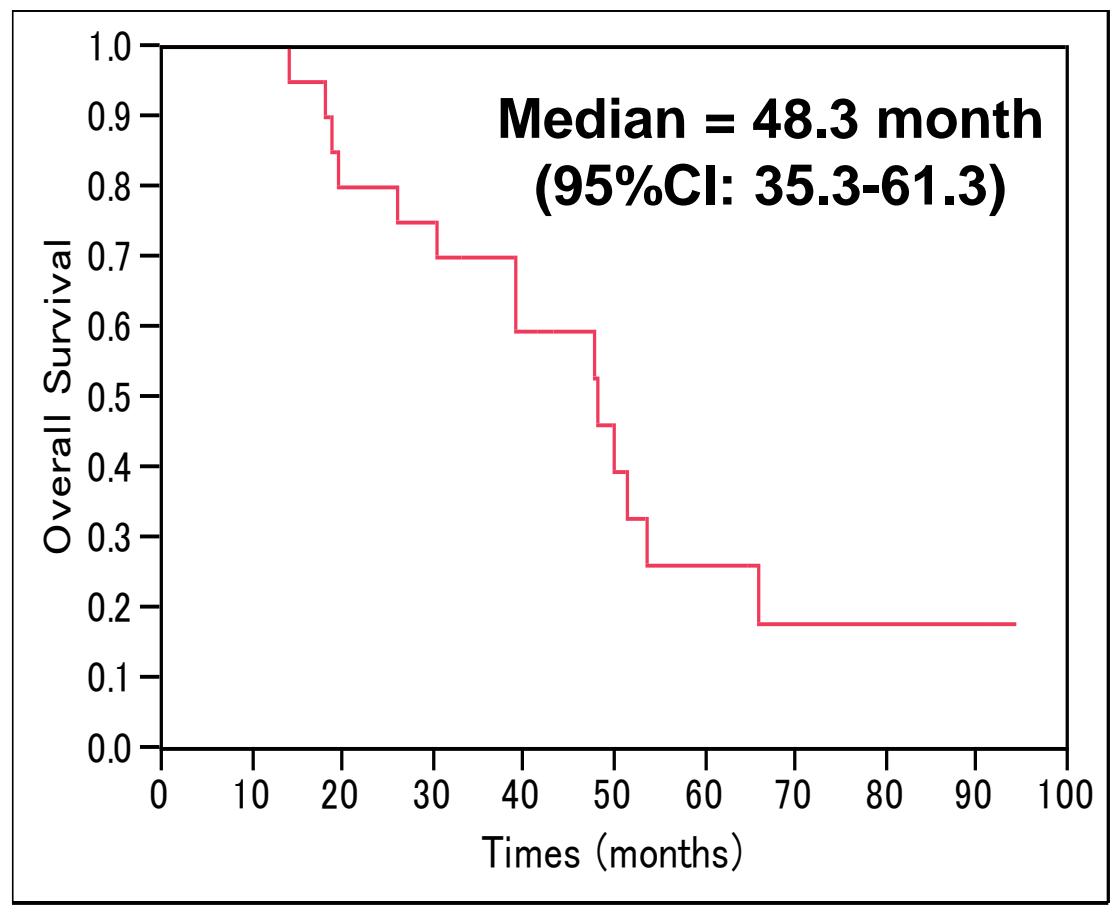

Figure 2 